\title{
Photoacoustic investigations on the photostability of Coumarin 540-doped PMMA
}

\author{
Ritty J. Nedumpara*, Binoy Paul, A. Santhi, P. Radhakrishnan, V.P.N. Nampoori \\ International School of Photonics, Cochin University of Science and Technology, Cochin 680222, Kerala, India
}

Received 21 May 2003; received in revised form 21 May 2003; accepted 7 June 2003

\begin{abstract}
In this paper, we present a laser-induced photoacoustic study on the photostability of laser dye Coumarin 540 doped in PMMA matrix and modified by the incorporation of low-molecular weight additives. The dependence of photostability of the dye on various experimental conditions, such as nature of solvents, incident optical power and dye concentration, is investigated in detail. The activation rates for the bleaching process are calculated for different concentrations and they suggest the possibility of two distinct mechanisms responsible for photodegradation. Further, analysis of the data confirms the linear dependence of photodegradation on the intensity of incident radiation. The role of different externally influencing parameters, such as wavelength and modulation frequency of incident radiation, is also discussed.

(C) 2003 Elsevier B.V. All rights reserved.
\end{abstract}

Keywords: Photostability; Dye-doped PMMA; Photoacoustic study

\section{Introduction}

The use of solid matrix for dye lasers is an attractive alternative to the conventional liquid dye solution due to its obvious advantages such as rigidity and compactness, lack of toxicity and solvent evaporation, absence of flow fluctuation, etc. They also have the commercial and technical advantages like low cost and safety of operation. Over the last decade, there has been renewed interest in the development of solid-state dye lasers. Various classes of materials like porous glasses, organically modified silicates, sol-gel materials and polymers were extensively studied as host materials because of their optical properties and commercial viability [1]. Among them, the simple organic polymer materials have emerged as the most promising ones. They possess many favorable optical properties in comparison with other type of solid hosts. The high homogeneity of this active medium is extremely important for narrow line width oscillators and for single-mode generation. They can quite effectively control the chemical composition at the formation stage $[2,3]$. In addition, they show much better compati-

\footnotetext{
* Corresponding author. Tel.: +91-484-2575848; fax: +91-484-2576714.

E-mail address: rtnedumpara@cusat.ac.in (R.J. Nedumpara).
}

bility with organic laser dyes, and the fabrication techniques are simple and inexpensive. Now, polymeric compositions incorporating organic laser dyes allow the design and development of compact laser systems and integrated optical systems [2]. The most frequently used polymeric material is polymethyl methacrylate (PMMA) because of its high optical transparency in the visible spectral range and resistance to laser damage [4].

In all applications of a laser dye, the main concern is the photochemical stability of the dye under irradiation by the pump light. While pumping with high-intensity beams at high repetition rates, many of the organic dyes showed a low power damage threshold of the matrices and thermal degradation or photodegradation of the dye [4]. Though laser dyes have been under use and investigations for a long time, many details of the processes responsible for dye stability and maintenance of the lasing properties against degradation are still vague [1]. The bleaching mechanism may vary from one class of dye to another and it may depend on the nature of the solvent, on the presence of other reactive impurities such as oxygen and also on the polymerization techniques. Recently, the main thrust in the development of solid-state dye lasers has been the search for suitable laser dyes and solid hosts that provide a high laser damage threshold and durability against photodegradation. 
The photoacoustic technique is found to be an effective tool for the optical and thermal characterization of various classes of materials. The acoustic wave generated by intensity-modulated optical illumination of the sample that is kept inside the photoacoustic cell is detected here. The resulting PA signal is a measure of the optical absorption coefficient of the sample. In this paper, we report the investigations on the nature of photodegradation of Coumarin 540 doped in PMMA matrix. We have employed PA method to analyze the photobleaching of the sample under intensity-modulated pulses of millisecond duration. The photostability is studied under different environments such as different solvents, varying optical powers, modulating frequencies, concentrations of the dye and pump wavelengths.

\section{Photostability investigations using PA technique}

Though the actual mechanism responsible for photostability is not fully known, it is essentially a photo-induced modification of the dye molecule. The photobleaching has shown a linear dependence on the incident intensity of radiation. The molecule excited by the absorption of radiation may return to the ground state unless it gets involved in a photochemical reaction or thermodestruction and loses its identity. The deexcitation may occur in a number of ways namely radiative emission such as fluorescence or phosphorescence, or non-radiative relaxation as thermal radiation. Whatever may be the process, the energy liberated will depend on the amount of energy absorbed by the molecules present in the material. If a photochemical reaction takes place, it will reduce the number of original dye molecules present in the material, which correspondingly changes the strength of emission. By measuring either the emission or absorption, one can estimate the amount of photodestruction taking place in the sample. In the present investigation, the non-radiative part of the deexcitation taking place in the sample is measured using PA technique.

From Rosenwaig-Gersho theory, the complex amplitude of PA signal generated by an optically transparent and thermally thick sample is given by $Q=\left(-\mathrm{i} \beta \mu^{2} \gamma P_{0} I_{0}\right) /$ $4 \sqrt{2} T_{0} l_{\mathrm{g}} a_{\mathrm{g}} k$, where $\beta$ is the absorption coefficient of the sample, $\gamma$ the ratio of specific heat capacities of air, $\mu$ the thermal diffusion length in the solid material, $P_{0}$ and $T_{0}$ the ambient pressure and temperature, $I_{0}$ the incident intensity of light, $l_{\mathrm{g}}$ the length of gas column inside the cavity, $a_{\mathrm{g}}$ the thermal diffusion length in the gas and $k$ the thermal conductivity of the sample [5]. Since this quantity is directly proportional to absorption coefficient $\beta$, any change in the number density of the dye molecule will result in a reduced absorption, which will in turn reduce the amplitude of PA signal. Thus, PA method can be adopted as an effective and easy way of investigating the photostability.

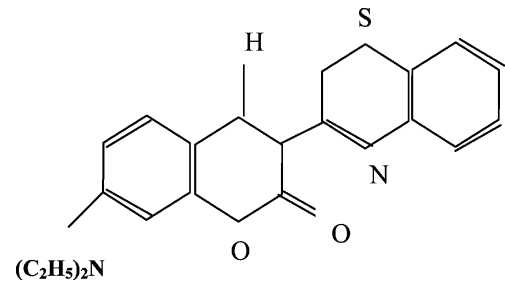

Fig. 1. Molecular structure of Coumarin 540 dye.

\section{Experimental details}

For the study of photostability of the Coumarin 540 dye-doped PMMA, samples are prepared under free radical polymerization. The dye is dissolved in a low-molecular weight additive and then mixed with the freshly prepared monomer MMA in a $1: 4(\mathrm{v} / \mathrm{v})$ ratio. The solution is filtered with a $0.6 \mu \mathrm{m}$ pore size paper (Whatman Div.). $0.3 \mathrm{~g} / \mathrm{l}$ AIBN is added as the initiator. The resulting solution is again filtered into cylindrical moulds and then they are sealed. Polymerization reaction is performed at $50{ }^{\circ} \mathrm{C}$ for 2 days and then at $45^{\circ} \mathrm{C}$ for 1 day [6,7]. The resulting cylindrical disks are cut and the surfaces are ground and polished for good optical quality.

The continuous wave $(\mathrm{cw})$ laser-induced photoacoustic technique is employed to investigate the photo-induced degradation of the Coumarin 540-doped PMMA samples (Fig. 1). The experimental setup used for the present study is schematically shown in Fig. 2. The source of light used is the Argon Ion Laser (Liconix 5400) and its different emission lines. The highly stabilized $\mathrm{cw}$ laser beam is intensity modulated using a mechanical chopper (Ithaco HMS 230). The modulated beam at specific power level is allowed to fall on the sample, which is kept in a non-resonant PA cell. A highly sensitive electret microphone (Knowles BT 1834) is used to detect PA signal in the reflection mode. PA signal is processed using a Digital lock-in amplifier (SR850 DSP). The thickness of the sample used for the study is of the order of $0.5 \mathrm{~mm}$.

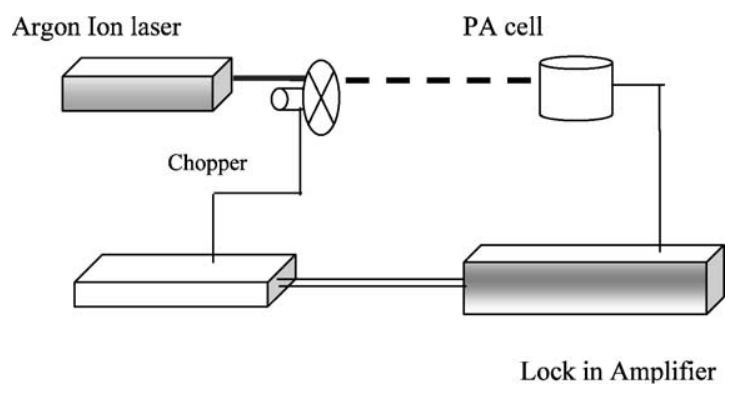

Fig. 2. Schematic diagram of the photoacoustic setup for the photostability studies. 


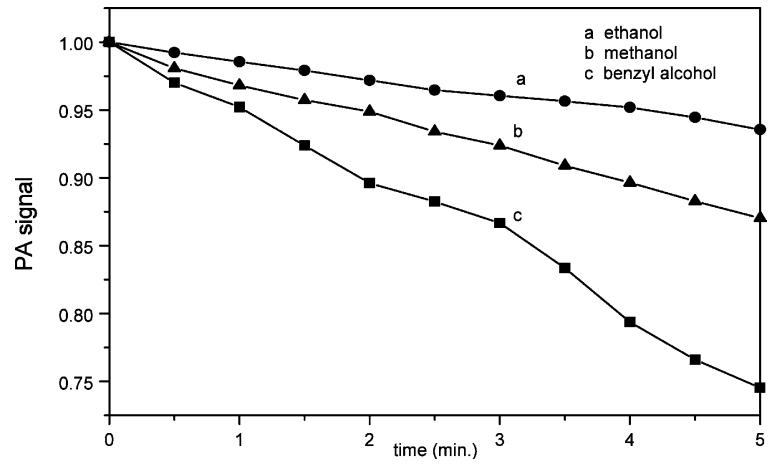

Fig. 3. PA signal amplitude versus time plot of Coumarin 540 doped PMMA for different solvents. (All values normalized with the corresponding maximum value.)

\section{Results and discussions}

The nature of PA signal variation with time has been studied under different environments. The photostability of the Coumarin 540-doped PMMA with different low-molecular weight additives is shown in Fig. 3. The dye has good solubility in all the three solvents. But the polymer matrix with ethanol as the solvent shows better stability though the difference is not very prominent. The photodestruction of the dye molecule is initiated by chemically active radicals of the basic polymer matrix. These radicals interact effectively with dye molecule at excited states and stimulate their destruction. The incorporation of a low-molecular weight additive is found to suppress the generation of these radical chains [8]. In this case, ethanol may be more effective in suppressing the generation of the radicals. The polymerization process also gives high-quality samples with ethanol. Thus, ethanol is selected as the solvent for all other investigations.

The effect of laser power on the stability of the dye-doped polymer matrix is investigated for different optical powers varying from 10 to $50 \mathrm{~mW}$. PA signal variation with different powers is as shown in Fig. 4. It is clear from the plot that bleaching rate increases with increased input light intensity. The fact that logarithmic plot of laser power versus bleaching rate (Fig. 5) gives a slope approximately equal to 1 shows that photobleaching is a linear process for incident light in-

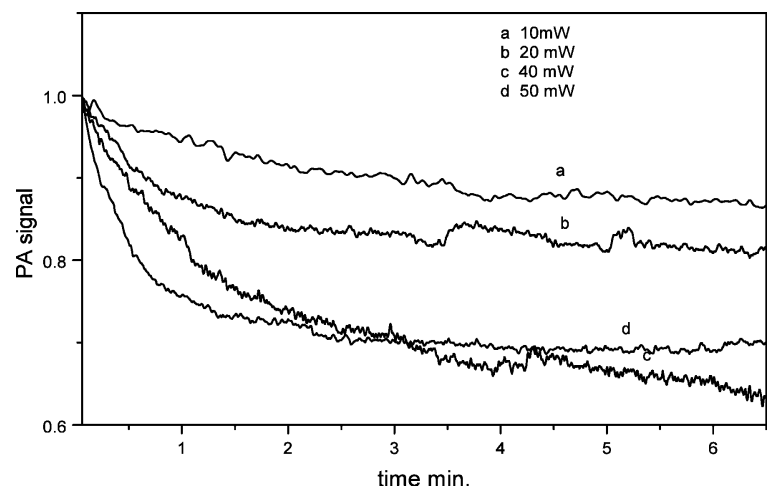

Fig. 4. PA signal amplitude versus time plot for different optical powers. (All values normalized with the corresponding maximum value.)

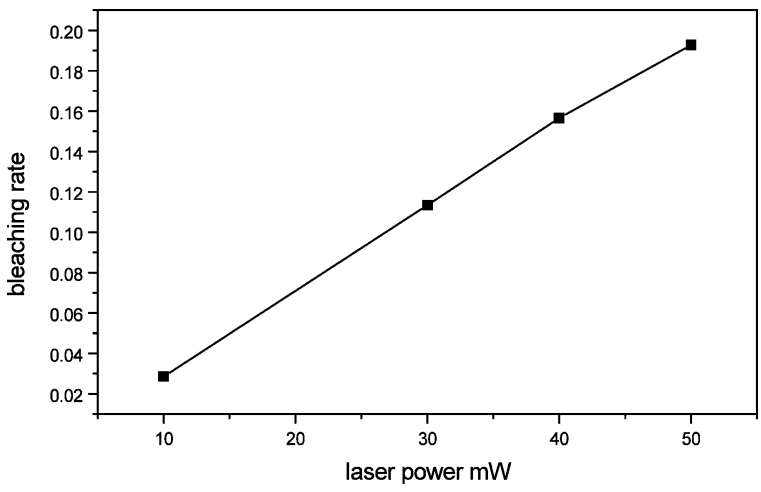

Fig. 5. Variation of rate of bleaching with incident laser power.

tensity due to a one-photon absorption. This is in agreement with the earlier reported studies by different authors [6,9]. This indicates that photobleaching in Coumarin 540-doped PMMA takes place when the system is in the first excited singlet state or triplet state and multiphoton absorption may not have any significant role in the photodegradation of the dye. The absorption spectrum of the sample recorded using a spectrophotometer before and after bleaching is shown in Fig. 6. This shows that photodestruction of the dye molecule takes place during irradiation of the sample and the resultant product does not absorb any radiation at $476 \mathrm{~nm}$ where a strong absorption exists for the original dye molecule. The decrease in PA signal is due to the photodegradation of the sample. The observed PA signal after the bleaching of the sample is contributed by the solid PMMA matrix. The photobleaching caused a color damage and the irradiated region became transparent. For higher input powers, the bleaching takes place at a faster rate.

The variation of photodestruction is studied for concentrations varying from $5 \times 10^{-3}$ to $1 \times 10^{-6} \mathrm{M} / \mathrm{l}$ and is shown in Fig. 7. We have used laser radiations of $30 \mathrm{~mW}$ power at a chopping frequency of $30 \mathrm{~Hz}$. In the lower concentration range, it is observed that photodestruction increases with decreasing concentrations of the dye, i.e. due to the reduced number density of the dye molecules [6]. But for very small concentrations like $1 \times 10^{-6} \mathrm{M} / \mathrm{l}$, PA signal showed

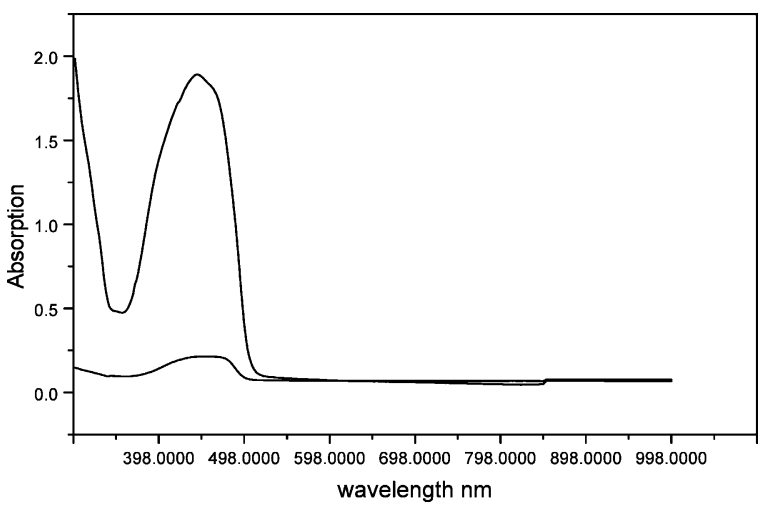

Fig. 6. Optical absorption spectrum of Coumarin 540 doped PMMA samples before and after bleaching. 


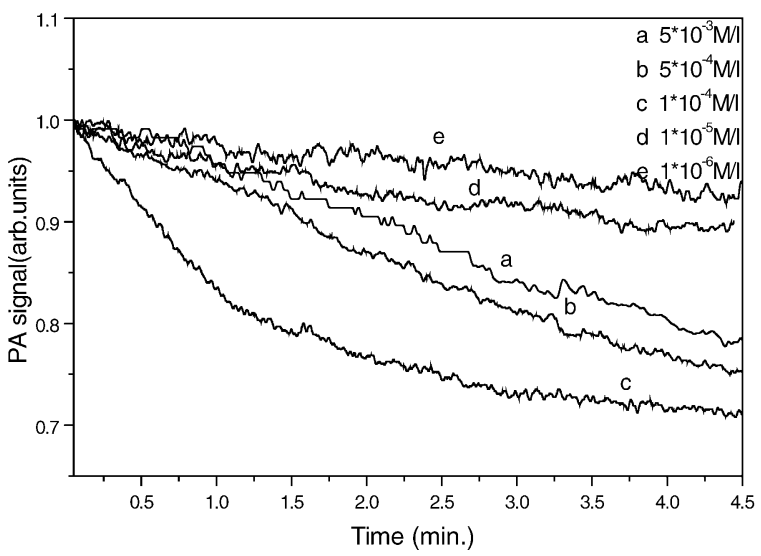

Fig. 7. PA signal amplitude versus time plot for different concentrations of the dye. (All values normalized with the corresponding maximum value.)

no considerable variation with time. At these concentrations, the activation time is very small so that the observed variation is very insignificant.

The influence of the modulating frequency on photostability has also been studied (Fig. 8). Though incident intensity is reduced with increased chopping rate, this has less effect on photodestruction which implies that the stability of the dye-doped polymer matrix depends on the total energy per unit time incident on the sample which is the same for all chopping frequencies. This is not the case for all dyes. A decrease in bleaching rate is observed with increased chopping rate in certain cases indicating the role of exposure time in the bleaching process [10].

The Coumarin 540 has two absorption bands, one in the short wavelength region and the other in the visible region [11]. The dependence of photodestruction on different wavelengths available from the argon ion laser shows that significant photobleaching is observed mainly for $476 \mathrm{~nm}$ (Fig. 9). Photobleaching is insensitive to radiation at wavelength of $514 \mathrm{~nm}$, which is in agreement with the absorption spectrum of the sample.

The main photodegradation mechanism should be a pho-

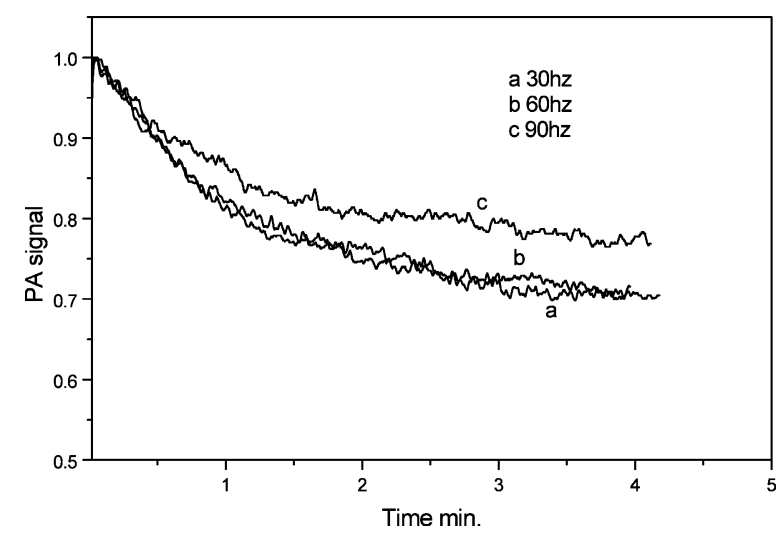

Fig. 8. PA signal amplitude versus time plot for different modulation frequencies. (All values normalized with the corresponding maximum value.)

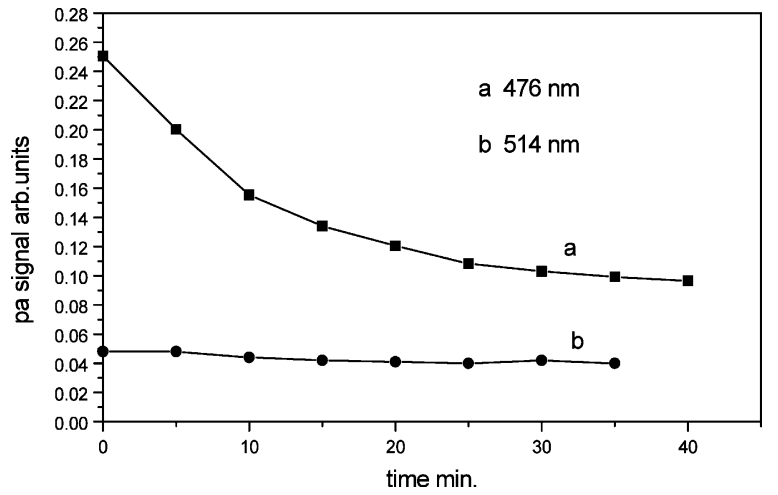

Fig. 9. PA signal amplitude versus time plot for different wavelengths.

Table 1

Photodegradation rates for different concentrations of the dye

\begin{tabular}{lll}
\hline Concentration of the dye $(\mathrm{M} / 1)$ & $1 / \tau_{1}\left(\mathrm{~ms}^{-1}\right)$ & $1 / \tau_{2}\left(\mathrm{~ms}^{-1}\right)$ \\
\hline $5 \times 10^{-3}$ & 0.217 & 0.664 \\
$5 \times 10^{-4}$ & 0.517 & 0.424 \\
$1 \times 10^{-4}$ & 1.35 & 0.462 \\
$1 \times 10^{-5}$ & 0.633 & 0.183 \\
$1 \times 10^{-6}$ & 0.301 & 0.142 \\
\hline
\end{tabular}

tochemical or photothermal process or both, but the specific contribution of each process is unknown [12]. The commonly accepted reasons for the degradation of dye molecules are thermodestruction due to the poor thermal dissipation in the polymer host, photodeactivation from the excited states which results in the transformation of the dye molecule, which gave the original absorption band of the dye, into components that no longer absorb light in that band and thirdly the formation of dimers that absorb irradiation without any fluorescence [1].

Assuming that the degradation rate of the dye obeys an exponential relation, PA signal amplitude can be written as $Q(t)=Q_{0} \exp (-t / \tau)$ at constant exposure to incident optical power. The degradation rate can be obtained from the slope of the curve plotted between time and $\log Q$. The decay rates at various concentrations of the dye are tabulated in Table 1. From the table, it is obvious that the photobleaching is a concentration-dependent process. Two distinct mechanisms are found to be prominent in the bleaching process. One is a faster process and the other a slower one. A more detailed investigation is going on to study the actual mechanism responsible for the photodegradation process.

\section{Conclusion}

The photostability of Coumarin 540-doped PMMA matrix under different environments is successfully investigated using PA technique. From experimental observations, it is found that ethanol is a good low-molecular weight additive for the Coumarin 540 dye doped in polymer matrix. It is established that the photodestruction of the dye is a lin- 
ear process with respect to incident light intensity. The dye destruction occurs from the first excited singlet or triplet electronic states. The bleaching rate linearly increases with the incident laser power. Bleaching is found to increase with decreasing concentrations of the dye molecule due to decreased optical density up to certain concentrations. No significant influence of the modulation frequency is observed on the bleaching rate. Prominent bleaching is observed only at $476 \mathrm{~nm}$ for all concentrations of the dye, as compared to the results for other longer wavelength of argon laser lines. The photodegradation rates are calculated for different concentrations of the dye, which shows the presence of two distinct mechanisms responsible for the photodegradation of the dye.

\section{Acknowledgements}

The authors gratefully acknowledge the financial support from the Netherlands University Federation for International Collaboration (NUFFIC).

\section{References}

[1] S. Popov, Appl. Opt. 37 (27) (1998) 6449.

[2] R. Sastre, A. Costela, Adv. Mater. 7 (2) (1995) 198.

[3] A. Costela, I. Garcia-Moreno, H. Tian, J. Su, K. Chen, F. Amat-Guerri, M. Carrascoso, J. Barroso, R. Sastre, Chem. Phys. Lett. 277 (1997) 392.

[4] G. Somasundaram, A. Ramalingam, Opt. Lasers Eng. 33 (2000) 157.

[5] A. Rosenwaig, A. Gersho, J. Appl. Phys. 47 (1) (1976) 64.

[6] A. George Nibu, B. Aneeshkumar, P. Radhakrishnan, C.P.G. Vallabhan, Appl. Phys. 32 (14) (1999) 1745.

[7] A. Costela, I. Garcia-Moreno, J.M. Figuera, F. Amat-Guerri, J. Barroso, R. Sastre, Opt. Commun. 130 (1996).

[8] K.M. Dyumaev, A.A. Manenkov, A.P. Maslyukov, G.A. Matyushin, V.S. Nechitailo, A.M. Prokhorov, Opt. Soc. Am. B 9 (1992) 143151.

[9] I.P. Kaminow, L.W. Stulz, E.A. Chandross, C.A. Pryde, Appl. Opt. 11 (7) (1972) 1563.

[10] A. Philip, P. Radhakrishnan, V.P.N. Nampoori, C.P.G. Vallabhan, Int. J. Opt. Elect. 8 (4) (1993) 501.

[11] E.P. Schafer (Ed.), Dye Lasers, Springer, Berlin, 1977.

[12] R. Duchowicz, L.B. Scaffardi, A. Costela, I. Garcia-Moreno, R. Sastre, A.U. Acuna, Appl. Opt. 39 (27) (2000) 4959. 